\title{
Competencia Sociolaboral en Relación al Nivel Formativo y Laboral del Alumnado de Formación Profesional de Ceuta
}

\section{Socio-Labor Competence Related with the Formative and Labor Level of the Vocational Education Students from Ceuta}

\author{
Lionel Sánchez-Bolívar ${ }^{1 *}$ y Sergio Escalante-González ${ }^{2}$ \\ 1 Departamento de Métodos de Investigación y Diagnóstico en Educación. Facultad de Ciencias de la Educación. Universidad de Granada (España). \\ 2 Facultad de Filosofía y Letras. Universidad de Granada. (España).
}

Resumen: Uno de los aspectos principales, durante el proceso de formación del alumnado y de su inserción en el mercado laboral, es el nivel de desarrollo social del mismo. Mediante este estudio, con un diseńo descriptivo, exploratorio y de corte transversal; se analizan las habilidades sociolaborales autopercibidas por el alumnado de formación profesional de un instituto de Ceuta. La muestra estaba formada por 100 individuos (86 mujeres y 14 hombres). El instrumento empleado fue el Test de factores sociopersonales para la inserción laboral de los jóvenes de Martínez-Rodríguez y Carmona (2010). En cuanto al nivel de estudios, el alumnado que procede de ciclos formativos de grado superior cuenta con mayor nivel de habilidades sociolaborales que aquel que accede con otras titulaciones. Respecto a la situación laboral del alumnado, quienes trabajan cuenta con mayores niveles de habilidades sociolaborales que los alumnos desempleados.

Palabras Clave: alumnado; competencia laboral; competencia social; formación profesional.
Abstract: One of the main aspects, during the student training process and its insertion in the labor market, is its level of social development. Through this study, with a descriptive, exploratory and cross-sectional design; the self-perceived socio-labor skills by the professional training students of a Ceuta institute are analyzed. The sample consisted of 100 individuals (86 women and $14 \mathrm{men}$ ). The instrument used was the Test of sociopersonal factors for the labor insertion of the young people of Martínez-Rodríguez \& Carmona (2010). Regarding the level of studies, students who come from higher cycles of vocational training have a higher level of socio-labor skills than students who access other degrees. Regarding the work situation of students, students who work have higher levels of socio-labor skills than unemployed students.

Key Words: students; labor competence; social competence; vocational training.

\section{Introducción}

Según "Eurostat" (2018), España es el segundo país con la mayor tasa de desempleo de la Unión Europea, sólo superado por Grecia, datos que se repiten respecto a la tasa de paro juvenil.

Uno de los principales problemas para acceder a un empleo es la falta de información acerca de los perfiles profesionales demandados por las empresas y los limitados conocimientos sobre los procesos de selección.

Tradicionalmente, la formación profesional era una salida laboral inmediata para aquel alumnado que carecía de interés por continuar con su vida académica, más allá de la formación básica, y a veces sin finalizar ésta.

En este sentido, Sánchez-Bolívar, Martínez-Martínez y Parra-González (2019) definen la formación profesional como "un sistema educativo con personalidad y currículo propios, en los que se aporta al alumnado que los cursa las competencias profesionales, personales y sociales necesarias para adquirir el rol de trabajador cualificado". (p.129)

Ahondando en la competencia profesional, como compe-

Dirección para correspondencia [Correspondence address]: Lionel Sánchez Bolívar. E-mail: lionelsanchez@correo.ugr.es tencia social fundamental ( Navarro-Patón, Lago-Ballesteros y Basanta-Camiño, 2019) y donde las habilidades sociolaborales van a tener un papel vertebrador, Martínez-Rodríguez y Carmona (2010), clasifican las habilidades sociolaborales, denominándolas cualidades personales, en: "imagen, empatía, control emocional, iniciativa, liderazgo, autonomía, ambición, atención y escucha, capacidad para la integración en el grupo (trabajo en equipo), cooperación y apoyo para el interés común, seguimiento de órdenes en la tarea y adaptaciones a situaciones cambiantes." (p.122)

En relación con lo expuesto, Reyna y Brussino (2015) exponen que el factor fundamental que impacta en el funcionamiento cognitivo y socio-emocional del alumnado es el nivel sociolaboral y económico, concretamente, de forma negativa, asociando un nivel socioeconómico bajo con problemas de conducta y emocionales.

Por todo esto, se ha planteado el presente estudio con los siguientes objetivos:

- Describir y analizar las variables: sexo, edad, situación laboral y nivel formativo.

- Relacionar las variables anteriormente citadas con las habilidades sociolaborales. 


\section{Material y método}

\section{Diseño}

Se llevó a cabo un estudio descriptivo, exploratorio y transversal.

\section{Participantes}

El muestreo se realizó con una población total de 100 alumnos $(\mathrm{N}=14)$ y alumnas $(\mathrm{N}=86)$ de formación profesional de grado medio y superior de las familias profesionales de Servicios a la Comunidad y de Electricidad y Electrónica de un Instituto de Educación Secundaria Obligatoria, Bachillerato y Formación Profesional de Ceuta, situado en un barrio de nivel socioeconómico medio-bajo.

\section{Variables e instrumentos}

Las variables sociodemográficas que se han definido para el estudio, y que se miden mediante un cuestionario ad-hoc, son: género (hombre o mujer); edad; situación laboral (trabajando o desempleado); formación o estudios realizados (no poseo el Título en Educación Secundaria Obligatoria -ESO-, Título en ESO o equivalente, Ciclo Formativo de Grado Medio -o equivalente-, Ciclo Formativo de Grado Superior -o equivalente-, Título de Bachillerato, Titulación universitaria -Diplomatura, Licenciatura, Grado, ...-).

Para la medición de las habilidades sociales se empleó el Test de factores sociopersonales para la inserción laboral de los jóvenes de Martínez-Rodríguez y Carmona (2010). El test consta de un total de 83 ítems, que, mediante una escala Likert de 1 a 10 , miden cuatro dimensiones: motivación para realizar la formación, satisfacción con la formación, cualidades para acceder a un empleo y valores personales. El tiempo estimado para su cumplimentación es de 25 minutos. El cuestionario arrojó una coeficiente de fiabilidad de $\alpha=, 961$ en este estudio.

\section{Procedimiento}

En primer lugar se contactó con el centro educativo, en marzo de 2018, para informar al equipo directivo acerca del estudio y las implicaciones que tendría para ellos. Con el visto bueno por parte de la directiva del centro, se procedió a seleccionar la muestra y pasar el cuestionario a los ciclos formativos, informando del anonimato del cuestionario y estando presentes los investigadores en todo momento para resolver cuantas dudas pudiese plantearles el mismo a los participantes.

\section{Análisis de los datos}

Para el análisis de los datos se utilizó el paquete estadístico software SPSS 20.0. En una primera parte se procede al análisis de los mismos haciendo uso de frecuencias, porcentajes y descriptivos como medias y desviaciones típicas y, en una segunda parte, se lleva a cabo un estudio relacional, empleando las pruebas paramétricas de $t$ de student para variables dicotómicas y el ANOVA de un factor para variables politómicas.

\section{Resultados}

La muestra, como se observa en la tabla 1, estaba compuesta por un $86 \%$ de mujeres $(\mathrm{N}=86)$ y un $14 \%(\mathrm{~N}=14)$ de hombres. Del total de la población, el 93\% ( $\mathrm{N}=93)$ se encontraba en situación de desempleo, mientras que el $7 \%(\mathrm{~N}=7)$ se encontraba trabajando.

Respecto a la edad, la población mayoritaria era de jóvenes entre 16 y 25 años, representando el 77\% (N=77) de la muestra.

En cuanto a la titulación, la tabla 2 refleja que el alumnado de formación profesional accede, principalmente, con el "Título de Bachillerato" (43\%; N=43) o con el "Título de Graduado en ESO” (31\%; N=31). 
Tabla 1. Distribución de la muestra y variables sociodemográficas.

\begin{tabular}{|c|c|c|c|c|c|c|c|c|c|c|c|}
\hline & & & $\mathrm{N}$ & $\%$ & & & & & & $\mathrm{~N}$ & $\%$ \\
\hline \multirow[t]{2}{*}{ Sexo } & Hombre & & 14 & $14 \%$ & & & Situación laboral & Empleado & & 7 & $7 \%$ \\
\hline & Mujer & & 86 & $86 \%$ & & & & Desempleado & & 93 & $93 \%$ \\
\hline \multirow[t]{10}{*}{ Edad } & 16 a 25 años & Hombre & 12 & $12 \%$ & 77 & $77 \%$ & Nivel de Estudios & ESO & Hombre & 7 & $7 \%$ \\
\hline & & Mujer & 65 & $65 \%$ & & & & & Mujer & 24 & $24 \%$ \\
\hline & 26 a 35 años & Hombre & 2 & $2 \%$ & 14 & $14 \%$ & & CFGM & Hombre & 1 & $1 \%$ \\
\hline & & Mujer & 12 & $12 \%$ & & & & & Mujer & 8 & $8 \%$ \\
\hline & 36 a 45 años & Hombre & 0 & $0 \%$ & 7 & $7 \%$ & & CFGS & Hombre & 0 & $0 \%$ \\
\hline & & Mujer & 7 & $7 \%$ & & & & & Mujer & 4 & $4 \%$ \\
\hline & 46 a 55 años & Hombre & 0 & $0 \%$ & 2 & $2 \%$ & & $\mathrm{BACH}$ & Hombre & 6 & $6 \%$ \\
\hline & & Mujer & 2 & $2 \%$ & & & & & Mujer & 37 & $37 \%$ \\
\hline & & & & & & & & TU & Hombre & 0 & $0 \%$ \\
\hline & & & & & & & & & Mujer & 13 & $13 \%$ \\
\hline
\end{tabular}

Nota: $\left({ }^{*}\right)$ ESO: Título en Educación Secundaria Obligatoria (ESO) o equivalente; CFGM: Ciclo Formativo de Grado Medio (o equivalente); CFGS: Ciclo Formativo de Grado Superior (o equivalente); BACH: Título de Bachillerato; TU: Titulación universitaria (Diplomatura, Licenciatura, Grado, etc.)

Atendiendo a la relación entre la "Formación de acceso" del alumnado y la primera dimensión "Motivos por los que realiza la formación" (tabla 2) no existen diferencias estadísticamente significativas $(\mathrm{p}>, 05)$ entre la formación de acceso del alumnado y ningún ítem relacionado con la motivación inicial por la que se matriculó en el ciclo de formación profesional que se encontraba cursando durante la investigación.

Tabla 2. Relación entre la Formación de Acceso y los Motivos por los que realiza la formación.

\begin{tabular}{|c|c|c|c|c|c|c|c|c|c|c|c|}
\hline & & $\mathrm{N}$ & Media & DT & Sig. & & & $\mathrm{N}$ & Media & DT & Sig. \\
\hline \multirow{5}{*}{$\begin{array}{l}\text { Tener título para } \\
\text { trabajar }\end{array}$} & ESO & 31 & 9,03 & 1,78 & & \multirow[t]{5}{*}{ Aumentar el currículum } & ESO & 31 & 8,9 & 1,60 & \\
\hline & CFGM & 9 & 9,67 & ,71 & & & CFGM & 9 & 9,11 & 1,69 & \\
\hline & CFGS & 4 & 8,75 & 2,50 & \multirow[t]{3}{*}{, 543} & & CFGS & 4 & 9,25 & ,96 &, 480 \\
\hline & $\mathrm{BACH}$ & 43 & 8,56 & 2,12 & & & BACH & 43 & 8,3 & 2,14 & \\
\hline & TU & 13 & 8,62 & 1,85 & & & TU & 13 & 9 & 1,53 & \\
\hline \multirow[t]{5}{*}{ Satisfacción personal } & ESO & 31 & 8,65 & 1,74 & & \multirow{5}{*}{$\begin{array}{l}\text { Tener más conocimientos } \\
\text { profesionales }\end{array}$} & ESO & 31 & 8,9 & 1,90 & \\
\hline & CFGM & 9 & 9,67 &, 50 & & & CFGM & 9 & 9,67 &, 71 & \\
\hline & CFGS & 4 & 10 & 0 &, 355 & & CFGS & 4 & 9,25 & 1,50 & ,708 \\
\hline & $\mathrm{BACH}$ & 43 & 8,88 & 1,66 & & & $\mathrm{BACH}$ & 43 & 9 & 1,50 & \\
\hline & TU & 13 & 8,85 & 2,03 & & & TU & 13 & 9,31 & 1,03 & \\
\hline \multirow{5}{*}{$\begin{array}{l}\text { Optar a una mejor } \\
\text { categoría profesional }\end{array}$} & ESO & 31 & 8,77 & 1,99 & & \multirow[t]{5}{*}{ Otros } & ESO & 31 & 8,06 & 2,93 & \\
\hline & CFGM & 9 & 9,89 & ,33 & & & CFGM & 9 & 8,78 & 2,17 & \\
\hline & CFGS & 4 & 10 & 0 & ,183 & & CFGS & 4 & 7,75 & 4,50 & ,739 \\
\hline & $\mathrm{BACH}$ & 43 & 8,84 & 1,86 & & & BACH & 43 & 7,47 & 2,87 & \\
\hline & TU & 13 & 8,15 & 2,11 & & & TU & 13 & 8,23 & 3,29 & \\
\hline \multirow[t]{5}{*}{ Encontrarse en paro } & ESO & 31 & 3,81 & 3,47 & & & & & & & \\
\hline & CFGM & 9 & 6,78 & 4,09 & & & & & & & \\
\hline & CFGS & 4 & 5,25 & 4,92 & 063 & & & & & & \\
\hline & $\mathrm{BACH}$ & 43 & 4,81 & 3,46 & & & & & & & \\
\hline & TU & 13 & 6,85 & 3,46 & & & & & & & \\
\hline
\end{tabular}

Nota: $\left({ }^{*}\right)$ ESO: Título en Educación Secundaria Obligatoria (ESO) o equivalente; CFGM: Ciclo Formativo de Grado Medio (o equivalente); CFGS: Ciclo Formativo de Grado Superior (o equivalente); BACH: Título de Bachillerato; TU: Titulación universitaria (Diplomatura, Licenciatura, Grado, etc.) 
En lo que respecta a la relación entre la "Formación de acceso" del alumnado y la segunda dimensión, "Satisfacción con la Formación”, la tabla 3 muestra que no se han detecta- do diferencias estadísticamente significativas ( $p>, 05)$ entre la formación de acceso y ninguno de los ítems que forman esta dimensión.

Tabla 3. Relación entre la Formación de acceso del alumnado y la Satisfacción con la Formación.

\begin{tabular}{|c|c|c|c|c|c|c|c|c|c|c|c|}
\hline & & $\mathrm{N}$ & Media & DT & Sig. & & & $\mathrm{N}$ & Media & DT & Sig. \\
\hline \multirow{5}{*}{$\begin{array}{l}\text { Con los métodos de } \\
\text { enseńanza }\end{array}$} & ESO & 31 & 8,06 & 2,34 & & \multirow{5}{*}{$\begin{array}{l}\text { Con la orientación } \\
\text { profesional }\end{array}$} & ESO & 31 & 7,32 & 2,70 & \\
\hline & CFGM & 9 & 9 & 1,32 & & & CFGM & 9 & 7,11 & 2,21 & \\
\hline & CFGS & 4 & 9,5 & 1,00 &, 392 & & CFGS & 4 & 8,25 & 2,36 & ,876 \\
\hline & BACH & 43 & 8,33 & 1,58 & & & BACH & 43 & 7,23 & 2,40 & \\
\hline & $\mathrm{TU}$ & 13 & 7,92 & 1,55 & & & $\mathrm{TU}$ & 13 & 6,77 & 2,20 & \\
\hline \multirow[t]{5}{*}{ Con el profesorado } & ESO & 31 & 7,32 & 2,47 & & \multirow{5}{*}{$\begin{array}{l}\text { Con la duración de } \\
\text { las prácticas }\end{array}$} & ESO & 31 & 6,9 & 3,04 & \multirow{5}{*}{,593 } \\
\hline & CFGM & 9 & 8,33 & 2,06 & & & CFGM & 9 & 7,89 & 1,69 & \\
\hline & CFGS & 4 & 9,25 & ,96 & \multirow[t]{3}{*}{, 251} & & CFGS & 4 & 7,75 & 2,06 & \\
\hline & BACH & 43 & 7,56 & 1,76 & & & BACH & 43 & 7,74 & 1,98 & \\
\hline & $\mathrm{TU}$ & 13 & 8,23 & 1,69 & & & $\mathrm{TU}$ & 13 & 7,31 & 1,89 & \\
\hline
\end{tabular}

Nota: $\left({ }^{*}\right)$ ESO: Título en Educación Secundaria Obligatoria (ESO) o equivalente; CFGM: Ciclo Formativo de Grado Medio (o equivalente); CFGS: Ciclo Formativo de Grado Superior (o equivalente); BACH: Título de Bachillerato; TU: Titulación universitaria (Diplomatura, Licenciatura, Grado, etc.)

Como se puede comprobar en la tabla 4, al relacionar la "Formación de acceso" con la dimensión "Cualidades Personales", se han hallado diferencias significativas a nivel estadístico $(\mathrm{p}<, 05)$ en la habilidad de "Disponibilidad" $(\mathrm{p}=, 030)$ para realizar acciones para el empleo y dentro del mismo, situándose los valores medios más altos en el alumnado procedente de ciclos formativos de grado superior $(\mathrm{M}=10,00, \mathrm{DT}=, 00)$.

Tabla 4. Relación entre la Formación de acceso del alumnado y las Cualidades Personales.

\begin{tabular}{|c|c|c|c|c|c|c|c|c|c|c|c|c|c|c|c|c|c|}
\hline & & $\mathrm{N}$ & $\mathrm{M}$ & DT & Sig. & & & $\mathrm{N}$ & $\mathrm{M}$ & DT & Sig. & & & $\mathrm{N}$ & $\mathrm{M}$ & DT & Sig. \\
\hline \multirow[t]{5}{*}{ IMG } & ESO & 31 & 8,19 & 1,96 & & CA & ESO & 31 & 8,97 & 1,64 & & HHLL & ESO & 31 & 7,55 & 2,05 & \\
\hline & CFGM & 9 & 8,44 & 1,74 & & & CFGM & 9 & 9,11 & 1,54 & & & CFGM & 9 & 7,33 & 1,66 & \\
\hline & CFGS & 4 & 8,75 & 2,50 & ,941 & & CFGS & 4 & 10,00 &, 00 & ,327 & & CFGS & 4 & 9,50 & 1,00 & ,079 \\
\hline & BACH & 43 & 8,16 & 1,99 & & & BACH & 43 & 8,65 & 1,45 & & & BACH & 43 & 8,26 & 1,43 & \\
\hline & $\mathrm{TU}$ & 13 & 8,54 & 1,27 & & & $\mathrm{TU}$ & 13 & 9,31 & 1,18 & & & $\mathrm{TU}$ & 13 & 8,15 & ,90 & \\
\hline \multirow[t]{5}{*}{ Empatía } & ESO & 31 & 8,68 & 1,78 & & SOT & ESO & 31 & 8,16 & 1,77 & & MOT & ESO & 31 & 8,23 & 1,61 & \\
\hline & CFGM & 9 & 9,78 & , 44 & & & CFGM & 9 & 8,89 & ,78 & & & CFGM & 9 & 8,44 & 1,24 & \\
\hline & CFGS & 4 & 9,75 &, 50 & ,254 & & CFGS & 4 & 9,25 & ,96 & ,052 & & CFGS & 4 & 9,50 & 1,00 & ,300 \\
\hline & BACH & 43 & 8,81 & 1,44 & & & BACH & 43 & 8,35 & 1,60 & & & BACH & 43 & 8,14 & 1,66 & \\
\hline & $\mathrm{TU}$ & 13 & 9,00 & 1,35 & & & $\mathrm{TU}$ & 13 & 9,54 & ,66 & & & $\mathrm{TU}$ & 13 & 8,92 & 1,38 & \\
\hline \multirow[t]{5}{*}{$\mathrm{CE}$} & ESO & 31 & 7,77 & 2,09 & & ASD & ESO & 31 & 7,87 & 2,11 & & AFT & ESO & 31 & 8,52 & 1,18 & \\
\hline & CFGM & 9 & 8,33 & 1,73 & & & CFGM & 9 & 8,44 & 1,59 & & & CFGM & 9 & 8,56 & 1,13 & \\
\hline & CFGS & 4 & 9,50 & 1,00 & , 407 & & CFGS & 4 & 8,25 & 2,22 & ,322 & & CFGS & 4 & 10,00 & ,00 & , 169 \\
\hline & BACH & 43 & 8,37 & 1,73 & & & BACH & 43 & 8,42 & 1,53 & & & BACH & 43 & 8,40 & 1,56 & \\
\hline & $\mathrm{TU}$ & 13 & 8,08 & 1,93 & & & $\mathrm{TU}$ & 13 & 9,08 & 1,26 & & & $\mathrm{TU}$ & 13 & 9,00 & 1,22 & \\
\hline \multirow[t]{5}{*}{ INI } & ESO & 31 & 8,58 & 2,08 & & CRN & ESO & 31 & 7,26 & 2,41 & & INT & ESO & 31 & 8,65 & 1,47 & \\
\hline & CFGM & 9 & 8,67 & 1,22 & & & CFGM & 9 & 6,89 & 1,62 & & & CFGM & 9 & 8,89 & 1,27 & \\
\hline & CFGS & 4 & 10,00 & ,00 & ,613 & & CFGS & 4 & 8,00 & 1,15 & ,362 & & CFGS & 4 & 9,75 &, 50 & ,345 \\
\hline & BACH & 43 & 8,58 & 1,61 & & & BACH & 43 & 7,95 & 1,57 & & & BACH & 43 & 8,65 & 1,34 & \\
\hline & $\mathrm{TU}$ & 13 & 8,46 & 1,71 & & & $\mathrm{TU}$ & 13 & 7,31 & 1,44 & & & $\mathrm{TU}$ & 13 & 9,23 & ,83 & \\
\hline
\end{tabular}




\begin{tabular}{|c|c|c|c|c|c|c|c|c|c|c|c|c|c|c|c|c|c|}
\hline & & $\mathrm{N}$ & $\mathrm{M}$ & DT & Sig. & & & $\mathrm{N}$ & $\mathrm{M}$ & DT & Sig. & & & $\mathrm{N}$ & $\mathrm{M}$ & DT & Sig. \\
\hline \multirow[t]{5}{*}{ LID } & ESO & 31 & 7,61 & 2,39 & & RA & ESO & 31 & 7,42 & 2,26 & & DIS & ESO & 31 & 8,58 & 1,41 & \\
\hline & CFGM & 9 & 8,22 & 1,72 & & & CFGM & 9 & 7,33 & 1,66 & & & CFGM & 9 & 8,22 & 1,64 & \\
\hline & CFGS & 4 & 9,50 & 1,00 & , 164 & & CFGS & 4 & 7,75 & 96 & ,385 & & CFGS & 4 & 10,00 &, 00 & ,030 \\
\hline & BACH & 43 & 7,86 & 1,98 & & & $\mathrm{BACH}$ & 43 & 8,02 & 1,57 & & & $\mathrm{BACH}$ & 43 & 9,28 & 1,03 & \\
\hline & TU & 13 & 6,69 & 2,29 & & & TU & 13 & 8,38 & 1,04 & & & TU & 13 & 8,92 & 1,50 & \\
\hline \multirow[t]{5}{*}{ AUT } & ESO & 31 & 8,03 & 2,40 & & CFV & ESO & 31 & 8,00 & 1,98 & & CRS & ESO & 31 & 8,42 & 1,82 & \\
\hline & CFGM & 9 & 9,22 & 1,09 & & & CFGM & 9 & 7,33 & 1,58 & & & CFGM & 9 & 8,89 & 1,36 & \\
\hline & CFGS & 4 & 9,50 & 1,00 & ,271 & & CFGS & 4 & 8,75 & 96 & ,303 & & CFGS & 4 & 10,00 &, 00 & ,423 \\
\hline & BACH & 43 & 8,70 & 1,39 & & & $\mathrm{BACH}$ & 43 & 8,44 & 1,33 & & & $\mathrm{BACH}$ & 43 & 8,58 & 1,55 & \\
\hline & TU & 13 & 8,85 & 2,19 & & & TU & 13 & 8,38 & 1,26 & & & TU & 13 & 8,85 & 1,63 & \\
\hline \multirow[t]{5}{*}{ AMB } & ESO & 31 & 7,97 & 1,83 & & CAN & ESO & 31 & 7,77 & 2,11 & & EAF & ESO & 31 & 8,77 & 1,87 & \\
\hline & CFGM & 9 & 8,22 & 2,39 & & & CFGM & 9 & 8,00 & 1,58 & & & CFGM & 9 & 9,22 & 97 & \\
\hline & CFGS & 4 & 9,00 & ,82 & ,402 & & CFGS & 4 & 8,25 &, 50 & 809 & & CFGS & 4 & 9,75 &, 50 & ,375 \\
\hline & BACH & 43 & 7,30 & 2,30 & & & $\mathrm{BACH}$ & 43 & 8,00 & 1,50 & & & $\mathrm{BACH}$ & 43 & 8,47 & 1,50 & \\
\hline & TU & 13 & 7,92 & 2,33 & & & TU & 13 & 8,46 & 1,45 & & & TU & 13 & 9,08 & 1,66 & \\
\hline \multirow[t]{5}{*}{$\mathrm{AE}$} & ESO & 31 & 8,87 & 1,80 & & CS & ESO & 31 & 7,68 & 2,44 & & ACE & ESO & 31 & 8,06 & 1,97 & \\
\hline & CFGM & 9 & 9,44 & 1,01 & & & CFGM & 9 & 7,56 & 1,33 & & & CFGM & 9 & 8,11 & 1,45 & \\
\hline & CFGS & 4 & 9,75 &, 50 & ,251 & & CFGS & 4 & 7,75 & 96 & ,780 & & CFGS & 4 & 9,50 & 1,00 & ,421 \\
\hline & BACH & 43 & 8,58 & 1,62 & & & $\mathrm{BACH}$ & 43 & 8,02 & 1,34 & & & $\mathrm{BACH}$ & 43 & 8,40 & 1,59 & \\
\hline & $\mathrm{TU}$ & 13 & 9,38 & ,87 & & & TU & 13 & 8,31 & 1,49 & & & TU & 13 & 8,77 & 1,17 & \\
\hline \multirow[t]{5}{*}{ CIG } & ESO & 31 & 8,81 & 1,76 & & $\mathrm{CON}$ & ESO & 31 & 7,06 & 2,29 & & RMLP & ESO & 31 & 7,26 & 1,91 & \\
\hline & CFGM & 9 & 9,00 & 1,32 & & & CFGM & 9 & 7,89 & 1,69 & & & CFGM & 9 & 8,56 & ,88 & \\
\hline & CFGS & 4 & 9,75 &, 50 & , 190 & & CFGS & 4 & 8,75 & 1,50 & ,177 & & CFGS & 4 & 9,25 & 96 &, 052 \\
\hline & BACH & 43 & 8,12 & 1,84 & & & BACH & 43 & 7,98 & 1,64 & & & $\mathrm{BACH}$ & 43 & 8,14 & 1,55 & \\
\hline & TU & 13 & 8,77 & 1,54 & & & TU & 13 & 7,92 & 95 & & & $\mathrm{TU}$ & 13 & 8,08 & 1,80 & \\
\hline
\end{tabular}

Nota: $\left(^{*}\right)$ ESO: Título en Educación Secundaria Obligatoria (ESO) o equivalente; CFGM: Ciclo Formativo de Grado Medio (o equivalente); CFGS: Ciclo Formativo de Grado Superior (o equivalente); BACH: Título de Bachillerato; TU: Titulación universitaria (Diplomatura, Licenciatura, Grado, etc.) (**) IMG: Imagen; CE: Control emocional; INI: Iniciativa; LID: Liderazgo; AUT: Autonomía; AMB: Ambición; AE: Atención y escucha; CIG: Capacidad para la integración en grupo; CA: Cooperación y apoyo; SOT: Seguir Órdenes en la Tarea; ASD: Adaptación a situaciones difíciles; CRN: Capacidad de razonamiento numérico; RA: Razonamiento abstracto; CFV: Capacidad y fluidez verbal; CAN: Capacidad de análisis; CS: Capacidad de síntesis; CON: Conocimientos; HHLL: Habilidades laborales; MOT: Motivación; AFT: Actitudes favorables al trabajo; INT: Interés; DIS: Disponibilidad; CRS: Competencia relacional social; EAF: Estabilidad y apoyo familiar; ACE: Adaptabilidad a la cultura de la empresa; RMLP: Relaciones en el seno del mercado laboral y profesional.

En cuanto a la "Formación del Alumnado" y la cuarta dimensión, "Valores Personales, como muestra la tabla 5, existen diferencias estadísticamente significativas $(\mathrm{p}<, 05)$ entre la "Formación del Alumnado" y el "Adorno" ( $\mathrm{p}=, 027)$-que hace referencia a la atención que el alumnado presta al aspecto físi- co y vestimenta-, contando el alumnado procedente de ciclos formativos de grado superior con valores medios superiores $(\mathrm{M}=8,00 ; \mathrm{DT}=2,83)$ al resto, mientras que el alumnado procedente de ESO cuenta con los valores más bajos $(\mathrm{M}=6,35$; $\mathrm{DT}=3,03)$.

Tabla 5. Relación entre Formación de acceso y Valores I.

\begin{tabular}{|c|c|c|c|c|c|c|c|c|c|c|c|}
\hline & & $\mathrm{N}$ & Media & DT & Sig. & & & $\mathrm{N}$ & Media & DT & Sig. \\
\hline \multirow[t]{5}{*}{ Sinceridad } & ESO & 31,00 & 8,81 & 1,74 & \multirow{5}{*}{,775 } & \multirow[t]{5}{*}{ Pensar } & ESO & 31 & 8,71 & 1,92 & \multirow{5}{*}{,779 } \\
\hline & CFGM & 9,00 & 8,67 & 1,32 & & & CFGM & 9 & 8,89 & 1,76 & \\
\hline & CFGS & 4,00 & 9,75 & 0,50 & & & CFGS & 4 & 9,75 & 0,50 & \\
\hline & BACH & 43,00 & 8,95 & 1,27 & & & BACH & 43 & 8,77 & 1,44 & \\
\hline & TU & 13,00 & 8,85 & 1,57 & & & $\mathrm{TU}$ & 13 & 9,00 & 1,08 & \\
\hline \multirow[t]{5}{*}{ Autosuperación } & ESO & 31,00 & 8,90 & 1,40 & \multirow{5}{*}{,388 } & \multirow[t]{5}{*}{ Saber } & ESO & 31 & 8,61 & 1,67 & \multirow{5}{*}{,263 } \\
\hline & CFGM & 9,00 & 9,67 & 0,50 & & & CFGM & 9 & 9,44 & 0,73 & \\
\hline & CFGS & 4,00 & 9,50 & 1,00 & & & CFGS & 4 & 10,00 & 0,00 & \\
\hline & BACH & 43,00 & 8,95 & 1,07 & & & BACH & 43 & 8,88 & 1,38 & \\
\hline & TU & 13,00 & 8,77 & 1,48 & & & $\mathrm{TU}$ & 13 & 8,85 & 0,99 & \\
\hline
\end{tabular}




\begin{tabular}{|c|c|c|c|c|c|c|c|c|c|c|c|}
\hline & & $\mathrm{N}$ & Media & DT & Sig. & & & $\mathrm{N}$ & Media & DT & Sig. \\
\hline \multirow[t]{5}{*}{ Iniciativa } & ESO & 31,00 & 9,13 & 1,36 & \multirow{5}{*}{, 817} & \multirow[t]{5}{*}{ Elegancia } & ESO & 31 & 8,19 & 1,74 & \multirow{5}{*}{,162 } \\
\hline & CFGM & 9,00 & 9,56 & 0,73 & & & CFGM & 9 & 7,89 & 2,03 & \\
\hline & CFGS & 4,00 & 9,50 & 1,00 & & & CFGS & 4 & 7,25 & 1,71 & \\
\hline & BACH & 43,00 & 9,00 & 1,50 & & & BACH & 43 & 8,35 & 1,62 & \\
\hline & $\mathrm{TU}$ & 13,00 & 9,08 & 1,38 & & & $\mathrm{TU}$ & 13 & 7,00 & 2,31 & \\
\hline \multirow[t]{5}{*}{ Independencia } & ESO & 31,00 & 8,45 & 1,63 & & \multirow[t]{5}{*}{ Estética } & ESO & 31 & 7,87 & 1,69 & \\
\hline & CFGM & 9,00 & 8,44 & 1,67 & & & CFGM & 9 & 7,44 & 2,55 & \\
\hline & CFGS & 4,00 & 10,00 & 0,00 & ,432 & & CFGS & 4 & 8,25 & 1,50 & ,831 \\
\hline & $\mathrm{BACH}$ & 43,00 & 8,51 & 1,72 & & & $\mathrm{BACH}$ & 43 & 8,16 & 1,63 & \\
\hline & TU & 13,00 & 8,92 & 1,61 & & & TU & 13 & 8,00 & 1,96 & \\
\hline \multirow{5}{*}{ Afectividad } & ESO & 31,00 & 8,10 & 1,64 & & \multirow{5}{*}{$\begin{array}{l}\text { Buena } \\
\text { imagen }\end{array}$} & ESO & 31 & 8,23 & 1,84 & \\
\hline & CFGM & 9,00 & 9,00 & 1,12 & & & CFGM & 9 & 7,78 & 2,54 & \\
\hline & CFGS & 4,00 & 9,75 & 0,50 &, 213 & & CFGS & 4 & 7,75 & 1,89 & ,832 \\
\hline & $\mathrm{BACH}$ & 43,00 & 8,05 & 1,94 & & & $\mathrm{BACH}$ & 43 & 8,47 & 1,64 & \\
\hline & $\mathrm{TU}$ & 13,00 & 7,77 & 2,17 & & & $\mathrm{TU}$ & 13 & 8,15 & 2,15 & \\
\hline \multirow[t]{5}{*}{ Emoción } & ESO & 31,00 & 7,90 & 1,72 & & \multirow[t]{5}{*}{ Adorno } & ESO & 31 & 6,35 & 3,03 & \\
\hline & CFGM & 9,00 & 9,00 & 1,22 & & & CFGM & 9 & 7,78 & 2,73 & \\
\hline & CFGS & 4,00 & 8,75 & 1,50 &, 257 & & CFGS & 4 & 8,00 & 2,83 & ,027 \\
\hline & BACH & 43,00 & 8,47 & 1,45 & & & BACH & 43 & 7,23 & 2,20 & \\
\hline & $\mathrm{TU}$ & 13,00 & 7,85 & 1,99 & & & TU & 13 & 4,69 & 3,47 & \\
\hline \multirow[t]{5}{*}{ Sentimiento } & ESO & 31,00 & 7,48 & 1,88 & & \multirow[t]{5}{*}{ Salud } & ESO & 31 & 8,65 & 2,01 & \\
\hline & CFGM & 9,00 & 8,67 & 1,73 & & & CFGM & 9 & 9,33 & 1,32 & \\
\hline & CFGS & 4,00 & 9,50 & 1,00 & ,091 & & CFGS & 4 & 9,25 & 0,96 & ,779 \\
\hline & BACH & 43,00 & 8,42 & 1,72 & & & BACH & 43 & 8,67 & 1,94 & \\
\hline & TU & 13,00 & 8,08 & 2,02 & & & TU & 13 & 9,08 & 1,19 & \\
\hline \multirow[t]{5}{*}{ Amor } & ESO & 31,00 & 7,03 & 3,03 & & \multirow{5}{*}{$\begin{array}{l}\text { Aseo perso- } \\
\text { nal }\end{array}$} & ESO & 31 & 9,16 & 1,24 & \\
\hline & CFGM & 9,00 & 7,67 & 2,69 & & & CFGM & 9 & 9,33 & 1,41 & \\
\hline & CFGS & 4,00 & 9,50 & 1,00 & ,409 & & CFGS & 4 & 9,75 & 0,50 &, 332 \\
\hline & BACH & 43,00 & 7,84 & 2,37 & & & BACH & 43 & 9,07 & 1,42 & \\
\hline & $\mathrm{TU}$ & 13,00 & 7,77 & 2,42 & & & TU & 13 & 9,85 & 0,38 & \\
\hline \multirow[t]{5}{*}{ Aprender } & ESO & 31,00 & 8,74 & 1,73 & & \multirow[t]{5}{*}{ Placer } & ESO & 31 & 8,35 & 1,85 & \\
\hline & CFGM & 9,00 & 9,89 & 0,33 & & & CFGM & 9 & 8,78 & 1,09 & \\
\hline & CFGS & 4,00 & 10,00 & 0,00 &, 237 & & CFGS & 4 & 9,00 & 0,82 & ,635 \\
\hline & BACH & 43,00 & 8,88 & 1,80 & & & BACH & 43 & 8,35 & 1,77 & \\
\hline & $\mathrm{TU}$ & 13,00 & 9,15 & 0,90 & & & $\mathrm{TU}$ & 13 & 7,69 & 2,66 & \\
\hline \multirow[t]{5}{*}{ Conocimiento } & ESO & 31,00 & 8,32 & 1,89 & & \multirow[t]{5}{*}{ Vitalidad } & ESO & 31 & 8,35 & 1,74 & \\
\hline & CFGM & 9,00 & 9,00 & 1,80 & & & CFGM & 9 & 9,11 & 1,27 & \\
\hline & CFGS & 4,00 & 9,75 & 0,50 & ,196 & & CFGS & 4 & 9,25 & 0,96 &, 354 \\
\hline & BACH & 43,00 & 9,00 & 1,11 & & & BACH & 43 & 8,91 & 1,34 & \\
\hline & TU & 13,00 & 9,00 & 1,22 & & & TU & 13 & 8,38 & 1,33 & \\
\hline
\end{tabular}

Nota: $\left({ }^{*}\right)$ ESO: Título en Educación Secundaria Obligatoria (ESO) o equivalente; CFGM: Ciclo Formativo de Grado Medio (o equivalente); CFGS: Ciclo Formativo de Grado Superior (o equivalente); BACH: Título de Bachillerato; TU: Titulación universitaria (Diplomatura, Licenciatura, Grado, etc.)

Así mismo, como refleja la tabla 6, se han encontrado diferencias estadísticamente significativas $(\mathrm{p}<, 05)$ entre la "Formación del Alumnado" y el ser "Tolerante" ( $\mathrm{p}=, 044)$, donde los valores medios más elevados se han hallado en el alumnado con titulación universitaria $(\mathrm{M}=9,31 ; \mathrm{DT}=, 85)$ y los más bajos en el alumnado con el título de ESO ( $\mathrm{M}=7,71$; $\mathrm{DT}=2,34)$. 
Tabla 6. Relación entre la Formación de Acceso del Alumnado y los Valores del mismo II.

\begin{tabular}{|c|c|c|c|c|c|c|c|c|c|c|c|}
\hline & & $\mathrm{N}$ & M & DT & Sig. & & & $\mathrm{N}$ & $\mathrm{M}$ & DT & Sig. \\
\hline \multirow[t]{5}{*}{ Fidelidad } & ESO & 31 & 8,55 & 1,61 & & Medioambiental & ESO & 31 & 7,58 & 2,09 & \\
\hline & CFGM & 9 & 8,78 & 1,64 & & & CFGM & 9 & 7,44 & 3,28 & \\
\hline & CFGS & 4 & 9,75 & 0,50 & ,628 & & CFGS & 4 & 8,75 & 0,96 & ,846 \\
\hline & $\mathrm{BACH}$ & 43 & 8,88 & 1,48 & & & BACH & 43 & 7,81 & 2,30 & \\
\hline & TU & 13 & 9,00 & 1,83 & & & TU & 13 & 8,08 & 2,06 & \\
\hline \multirow[t]{5}{*}{ Obediencia } & ESO & 31 & 8,48 & 1,82 & & Desarrollo sostenible & ESO & 31 & 7,77 & 2,03 & \\
\hline & CFGM & 9 & 8,33 & 2,50 & & & CFGM & 9 & 7,56 & 3,05 & \\
\hline & CFGS & 4 & 8,25 & 2,22 & ,972 & & CFGS & 4 & 9,25 & 0,96 & ,689 \\
\hline & $\mathrm{BACH}$ & 43 & 8,67 & 1,63 & & & BACH & 43 & 7,93 & 2,11 & \\
\hline & TU & 13 & 8,46 & 1,76 & & & TU & 13 & 8,23 & 1,83 & \\
\hline \multirow[t]{5}{*}{ Honradez } & ESO & 31 & 9,00 & 1,26 & & Trabajo & ESO & 31 & 9,23 & 1,33 & \\
\hline & CFGM & 9 & 9,56 & 0,73 & & & CFGM & 9 & 9,89 & 0,33 & \\
\hline & CFGS & 4 & 10,00 & 0,00 & ,496 & & CFGS & 4 & 10,00 & 0,00 & , 147 \\
\hline & $\mathrm{BACH}$ & 43 & 9,23 & 1,44 & & & BACH & 43 & 8,77 & 1,72 & \\
\hline & TU & 13 & 9,38 & 0,77 & & & TU & 13 & 8,92 & 1,26 & \\
\hline \multirow[t]{5}{*}{ Responsabilidad } & ESO & 31 & 9,16 & 1,37 & & Ahorro & ESO & 31 & 8,23 & 2,53 & \\
\hline & CFGM & 9 & 9,44 & 1,01 & & & CFGM & 9 & 8,33 & 1,80 & \\
\hline & CFGS & 4 & 10,00 & 0,00 &, 500 & & CFGS & 4 & 8,75 & 0,96 & ,832 \\
\hline & $\mathrm{BACH}$ & 43 & 9,35 & 1,33 & & & BACH & 43 & 8,33 & 2,06 & \\
\hline & $\mathrm{TU}$ & 13 & 9,77 & 0,60 & & & $\mathrm{TU}$ & 13 & 7,62 & 1,33 & \\
\hline \multirow[t]{5}{*}{ Convivencia } & ESO & 31 & 9,35 & 0,98 & & Dinero & ESO & 31 & 8,26 & 1,93 & \\
\hline & CFGM & 9 & 9,44 & 1,33 & & & CFGM & 9 & 9,11 & 1,62 & \\
\hline & CFGS & 4 & 10,00 & 0,00 & ,616 & & CFGS & 4 & 9,25 & 0,96 & ,098 \\
\hline & $\mathrm{BACH}$ & 43 & 9,07 & 1,58 & & & BACH & 43 & 8,21 & 1,54 & \\
\hline & $\mathrm{TU}$ & 13 & 9,15 & 0,90 & & & TU & 13 & 7,31 & 1,49 & \\
\hline \multirow[t]{5}{*}{ Colaboración } & ESO & 31 & 9,48 & 0,89 & & Sueldo & ESO & 31 & 8,03 & 2,66 & \\
\hline & CFGM & 9 & 9,89 & 0,33 & & & CFGM & 9 & 8,78 & 1,92 & \\
\hline & CFGS & 4 & 10,00 & 0,00 & ,302 & & CFGS & 4 & 9,75 & 0,50 &, 528 \\
\hline & $\mathrm{BACH}$ & 43 & 9,28 & 1,10 & & & $\mathrm{BACH}$ & 43 & 8,37 & 1,90 & \\
\hline & TU & 13 & 9,38 & 0,77 & & & $\mathrm{TU}$ & 13 & 7,92 & 1,89 & \\
\hline \multirow[t]{5}{*}{ Tolerante } & ESO & 31 & 7,71 & 2,34 & & Creyente & ESO & 31 & 6,03 & 3,75 & \\
\hline & CFGM & 9 & 8,56 & 1,59 & & & CFGM & 9 & 6,67 & 4,12 & \\
\hline & CFGS & 4 & 8,50 & 1,00 &, 044 & & CFGS & 4 & 8,75 & 2,50 & ,396 \\
\hline & $\mathrm{BACH}$ & 43 & 8,74 & 1,43 & & & BACH & 43 & 6,60 & 3,32 & \\
\hline & $\mathrm{TU}$ & 13 & 9,31 & 0,85 & & & $\mathrm{TU}$ & 13 & 5,00 & 3,98 & \\
\hline \multirow[t]{5}{*}{ Participación } & ESO & 31 & 8,81 & 1,85 & & Ateo & ESO & 31 & 4,16 & 3,65 & \\
\hline & CFGM & 9 & 8,78 & 1,56 & & & CFGM & 9 & 2,56 & 3,09 & \\
\hline & CFGS & 4 & 10,00 & 0,00 & ,467 & & CFGS & 4 & 4,25 & 4,27 & ,257 \\
\hline & $\mathrm{BACH}$ & 43 & 8,77 & 1,80 & & & BACH & 43 & 5,33 & 3,51 & \\
\hline & TU & 13 & 9,46 & 0,78 & & & $\mathrm{TU}$ & 13 & 4,00 & 3,96 & \\
\hline
\end{tabular}




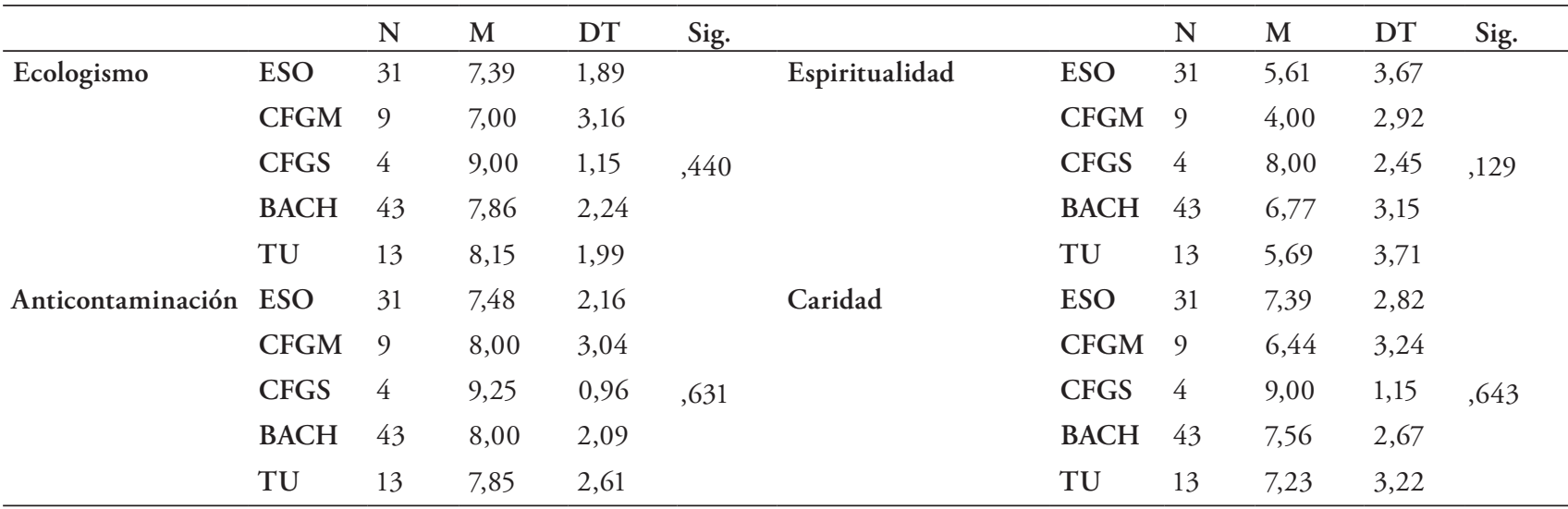

Nota: $\left(^{*}\right)$ ESO: Título en Educación Secundaria Obligatoria (ESO) o equivalente; CFGM: Ciclo Formativo de Grado Medio (o equivalente); CFGS: Ciclo Formativo de Grado Superior (o equivalente); BACH: Título de Bachillerato; TU: Titulación universitaria (Diplomatura, Licenciatura, Grado, etc.)

Por otro lado observamos en la tabla 7 que no se han iden- tre la "situación laboral" del alumnado y el "motivo de acceso tificado diferencias estadísticamente (p>,05) significativas en- a la formación”.

Tabla 7. Relación entre la situación laboral y los motivos de acceso a la formación.

\begin{tabular}{|c|c|c|c|c|c|}
\hline & & $\mathrm{N}$ & Media & DT & Sig. \\
\hline \multirow{2}{*}{ Tener título para trabajar } & Desempleado/a & 93 & 8,86 & 1,87 & \multirow{2}{*}{,444 } \\
\hline & Trabajando & 7 & 8,29 & 2,36 & \\
\hline \multirow[t]{2}{*}{ Satisfacción personal } & Desempleado/a & 93 & 8,96 & 1,57 & \multirow{2}{*}{,626 } \\
\hline & Trabajando & 7 & 8,43 & 2,70 & \\
\hline \multirow[t]{2}{*}{ Optar a una mejor categoría profesional } & Desempleado/a & 93 & 8,89 & 1,83 & \multirow{2}{*}{,661 } \\
\hline & Trabajando & 7 & 8,57 & 2,30 & \\
\hline \multirow[t]{2}{*}{ Encontrarse en paro } & Desempleado/a & 93 & 4,90 & 3,67 & \multirow{2}{*}{, 575} \\
\hline & Trabajando & 7 & 5,71 & 3,82 & \\
\hline \multirow[t]{2}{*}{ Aumentar el currículum } & Desempleado/a & 93 & 8,63 & 1,89 & \multirow{2}{*}{,273 } \\
\hline & Trabajando & 7 & 9,43 &, 79 & \\
\hline \multirow[t]{2}{*}{ Tener más conocimientos profesionales } & Desempleado/a & 93 & 9,06 & 1,55 & \multirow{2}{*}{,714 } \\
\hline & Trabajando & 7 & 9,29 & 1,25 & \\
\hline \multirow[t]{2}{*}{ Otros } & Desempleado/a & 93 & 7,82 & 2,90 & \multirow{2}{*}{,438 } \\
\hline & Trabajando & 7 & 8,71 & 3,40 & \\
\hline
\end{tabular}

Nota: elaboración propia.

Respecto a la "situación laboral" del alumnado y su relación con la "satisfacción" del mismo, tal y como se observa en la tabla 8 , se han hallado diferencias significativas a nivel estadístico $(\mathrm{p}<, 05)$ entre la "situación laboral" del alumna- do y la satisfacción "con los métodos de enseńanza" ( $\mathrm{p}=, 009)$, mostrándose el alumnado en situación de empleo activo con valores medios de satisfacción $(M=9,29 ; \mathrm{DT}=, 76)$ que el alumnado "desempleado" (M=8,23; DT=1,86). 
Tabla 8. Relación entre la Situación laboral y la Satisfacción del alumnado.

\begin{tabular}{|c|c|c|c|c|c|}
\hline & & $\mathrm{N}$ & Media & DT & Sig. \\
\hline \multirow[t]{2}{*}{ Con los métodos de enseńanza } & Desempleado/a & 93 & 8,23 & 1,86 & \multirow{2}{*}{,009 } \\
\hline & Trabajando & 7 & 9,29 &, 76 & \\
\hline \multirow[t]{2}{*}{ Con el Profesorado } & Desempleado/a & 93 & 7,58 & 2,03 & \multirow{2}{*}{, 000} \\
\hline & Trabajando & 7 & 9,43 &, 79 & \\
\hline \multirow[t]{2}{*}{ Con la orientación profesional } & Desempleado/a & 93 & 7,23 & 2,39 & \multirow{2}{*}{,950 } \\
\hline & Trabajando & 7 & 7,29 & 3,04 & \\
\hline \multirow[t]{2}{*}{ Con la duración de las prácticas } & Desempleado/a & 93 & 7,45 & 2,36 & \multirow{2}{*}{,857 } \\
\hline & Trabajando & 7 & 7,29 & 1,89 & \\
\hline
\end{tabular}

Nota: elaboración propia

Asimismo, la tabla 8 muestra que existen diferencias estadísticamente significativas $(\mathrm{p}<, 05)$ entre la "situación laboral del alumnado" y su satisfacción "con el profesorado" $(\mathrm{p}=, 000)$, situándose los valores medios más elevados en el alumnado que está "trabajando" ( $M=9,43 ; \mathrm{DT}=, 79)$, existiendo una diferencia de medias de casi dos puntos respecto del alumnado "desempleado" ( $M=7,58 ; \mathrm{DT}=2,03)$.
Como se puede comprobar en la tabla 9 , se han identificado diferencias estadísticamente significativas $(p<, 05)$ entre la "situación laboral" del alumnado y el "seguimiento de órdenes en la tarea" $(\mathrm{p}=, 000)$, contando el alumnado "desempleado" con niveles inferiores de esta habilidad $(M=8,44 ; \mathrm{DT}=1,56)$ que el alumnado en situación activa $(M=9,71 ; \mathrm{DT}=, 49)$.

Tabla 9. Relación entre la situación laboral y las cualidades del alumnado.

\begin{tabular}{|c|c|c|c|c|c|}
\hline & & $\mathrm{N}$ & Media & DT & Sig. \\
\hline \multirow[t]{2}{*}{ Imagen } & Desempleado/a & 93 & 8,24 & 1,89 & \multirow{2}{*}{, 517} \\
\hline & Trabajando & 7 & 8,71 & 1,60 & \\
\hline \multirow[t]{2}{*}{ Empatía } & Desempleado/a & 93 & 8,88 & 1,52 & \multirow{2}{*}{,349 } \\
\hline & Trabajando & 7 & 9,43 &, 53 & \\
\hline \multirow[t]{2}{*}{ Control emocional } & Desempleado/a & 93 & 8,18 & 1,88 & \multirow{2}{*}{,889 } \\
\hline & Trabajando & 7 & 8,29 & 1,80 & \\
\hline \multirow[t]{2}{*}{ Iniciativa } & Desempleado/a & 93 & 8,59 & 1,74 & \multirow{2}{*}{,416 } \\
\hline & Trabajando & 7 & 9,14 & 1,46 & \\
\hline \multirow[t]{2}{*}{ Liderazgo } & Desempleado/a & 93 & 7,68 & 2,17 & \multirow{2}{*}{,374 } \\
\hline & Trabajando & 7 & 8,43 & 1,81 & \\
\hline \multirow[t]{2}{*}{ Autonomía } & Desempleado/a & 93 & 8,53 & 1,90 & \multirow{2}{*}{, 218} \\
\hline & Trabajando & 7 & 9,43 & ,79 & \\
\hline \multirow[t]{2}{*}{ Ambición } & Desempleado/a & 93 & 7,71 & 2,18 & \multirow{2}{*}{,609 } \\
\hline & Trabajando & 7 & 8,14 & 1,57 & \\
\hline \multirow[t]{2}{*}{ Atención y escucha } & Desempleado/a & 93 & 8,87 & 1,60 & \multirow{2}{*}{,499 } \\
\hline & Trabajando & 7 & 9,29 &, 76 & \\
\hline \multirow[t]{2}{*}{ Capacidad para la integración en grupo } & Desempleado/a & 93 & 8,52 & 1,75 & \multirow{2}{*}{,358 } \\
\hline & Trabajando & 7 & 9,14 & 1,46 & \\
\hline \multirow[t]{2}{*}{ Cooperación y apoyo } & Desempleado/a & 93 & 8,88 & 1,49 & \multirow{2}{*}{,234 } \\
\hline & Trabajando & 7 & 9,57 & 1,13 & \\
\hline \multirow[t]{2}{*}{ Seguimiento de órdenes en la tarea } & Desempleado/a & 93 & 8,44 & 1,56 & \multirow{2}{*}{, 000} \\
\hline & Trabajando & 7 & 9,71 & ,49 & \\
\hline \multirow[t]{2}{*}{ Adaptación a situaciones difíciles } & Desempleado/a & 93 & 8,27 & 1,75 & \multirow{2}{*}{,202 } \\
\hline & Trabajando & 7 & 9,14 & 1,46 & \\
\hline \multirow[t]{2}{*}{ Capacidad de razonamiento numérico } & Desempleado/a & 93 & 7,48 & 1,89 & \multirow{2}{*}{,027 } \\
\hline & Trabajando & 7 & 8,57 & ,98 & \\
\hline
\end{tabular}




\begin{tabular}{|c|c|c|c|c|c|}
\hline & & $\mathrm{N}$ & Media & DT & Sig. \\
\hline \multirow[t]{2}{*}{ Razonamiento abstracto } & Desempleado/a & 93 & 7,75 & 1,81 & \multirow{2}{*}{,008 } \\
\hline & Trabajando & 7 & 8,57 &, 53 & \\
\hline \multirow[t]{2}{*}{ Capacidad y fluidez verbal } & Desempleado/a & 93 & 8,17 & 1,59 & \multirow{2}{*}{,383 } \\
\hline & Trabajando & 7 & 8,71 & 1,38 & \\
\hline \multirow[t]{2}{*}{ Capacidad de análisis } & Desempleado/a & 93 & 7,91 & 1,70 & \multirow{2}{*}{,002 } \\
\hline & Trabajando & 7 & 9,14 & ,69 & \\
\hline \multirow[t]{2}{*}{ Capacidad de síntesis } & Desempleado/a & 93 & 7,82 & 1,77 & \multirow{2}{*}{,020 } \\
\hline & Trabajando & 7 & 9,00 & 1,00 & \\
\hline \multirow[t]{2}{*}{ Conocimientos (teóricos) técnicos-profesionales } & Desempleado/a & 93 & 7,66 & 1,88 & \multirow{2}{*}{, 050} \\
\hline & Trabajando & 7 & 8,43 & ,79 & \\
\hline \multirow[t]{2}{*}{ Habilidades laborales (destrezas y resolución práctica) } & Desempleado/a & 93 & 7,92 & 1,67 & \multirow{2}{*}{, 150} \\
\hline & Trabajando & 7 & 8,86 & 1,07 & \\
\hline \multirow{2}{*}{$\begin{array}{l}\text { Motivación (causalidad interna del sujeto que le impulsa a } \\
\text { desempeñar la actividad laboral) }\end{array}$} & Desempleado/a & 93 & 8,33 & 1,56 & \multirow{2}{*}{,700 } \\
\hline & Trabajando & 7 & 8,57 & 1,72 & \\
\hline \multirow[t]{2}{*}{ Actitudes favorables al trabajo (dedicación, constancia, etc.) } & Desempleado/a & 93 & 8,55 & 1,38 & \multirow{2}{*}{,268 } \\
\hline & Trabajando & 7 & 9,14 & 1,07 & \\
\hline \multirow[t]{2}{*}{ Interés (atracción sentida por el aspirante hacia el empleo) } & Desempleado/a & 93 & 8,76 & 1,35 & \multirow{2}{*}{,464 } \\
\hline & Trabajando & 7 & 9,14 & ,69 & \\
\hline \multirow[t]{2}{*}{ Disponibilidad } & Desempleado/a & 93 & 8,98 & 1,27 & \multirow{2}{*}{,432 } \\
\hline & Trabajando & 7 & 8,57 & 1,90 & \\
\hline \multirow{2}{*}{$\begin{array}{l}\text { Competencia relacional social (capacidad para relacionarse en } \\
\text { distintos ámbitos y estamentos sociales) }\end{array}$} & Desempleado/a & 93 & 8,66 & 1,58 & \multirow{2}{*}{,894 } \\
\hline & Trabajando & 7 & 8,57 & 2,15 & \\
\hline \multirow[t]{2}{*}{ Estabilidad y apoyo familiar } & Desempleado/a & 93 & 8,72 & 1,60 & \multirow{2}{*}{, 369} \\
\hline & Trabajando & 7 & 9,29 & 1,50 & \\
\hline \multirow[t]{2}{*}{ Adaptabilidad a la cultura de la empresa } & Desempleado/a & 93 & 8,33 & 1,68 & \multirow{2}{*}{, 558} \\
\hline & Trabajando & 7 & 8,71 & 1,25 & \\
\hline \multirow[t]{2}{*}{ Relaciones en el seno del mercado laboral y profesional } & Desempleado/a & 93 & 7,95 & 1,68 & \multirow{2}{*}{,894 } \\
\hline & Trabajando & 7 & 7,86 & 2,12 & \\
\hline
\end{tabular}

Nota: elaboración propia

También se han hallado diferencias estadísticamente significativas $(\mathrm{p}<, 05)$ entre la "situación laboral" y la "capacidad de razonamiento numérico" ( $\mathrm{p}=, 027)$, contando el alumnado empleado $(\mathrm{M}=8,57$; $\mathrm{DT}=, 98)$ con niveles medios superiores en esta habilidad, respecto al alumnado "desempleado".

En cuanto a la "capacidad de análisis" y la "situación laboral", existen diferencias significativas a nivel estadístico $(\mathrm{p}=, 002)$, situándose los valores medios más elevados en el alumnado que se encuentra "trabajando" (M=9,14; DT=,69).

Respecto a la relación entre la "situación laboral" del alumnado y la "capacidad de síntesis", la tabla 9 muestra que existen diferencias estadísticamente significativas $(\mathrm{p}<, 05)$ entre ambas $(\mathrm{p}=, 020)$, contando los "desempleados" con valores medios inferiores $(\mathrm{M}=7,82 ; \mathrm{DT}=1,77)$ a los del alumnado que trabaja $(\mathrm{M}=9,00 ; \mathrm{DT}=1,00)$.

\section{Discusión}

El propósito de este estudio es analizar la competencia laboral del alumnado matriculado en títulos de formación profesio- nal en un instituto de Ceuta, así como evaluar las diferencias en el nivel de esta competencia según la titulación con la que accedieron a la formación profesional y a su estatus laboral.

En lo que se refiere a la titulación de acceso, el alumnado que accedió con un ciclo formativo de grado superior manifiesta una mayor disponibilidad formativa y laboral. En el polo opuesto se encuentra el alumnado que accedió con titulaciones de inferior rango como los ciclos formativos de grado medio o la ESO. En este sentido, el alumnado que accedió a formación profesional tras cursar estudios universitarios cuenta con niveles superiores de tolerancia, asertividad y empatía que el alumnado que accedió con titulaciones de menor nivel académico.

Estos resultados coinciden con los obtenidos por Reyna y Brussino (2015), que concluyen que conforme se avanza en edad y etapa educativa hay un mayor nivel de habilidad social y una mayor disponibilidad formativa. Asimismo, se contraponen a lo expuesto por autores como Serrano-Moras y Marín-rodas (2015), cuyos resultados apuntaban a una menor habilidad social en alumnado de mayor nivel académico, 
manifestando actitudes más agresivas y desafiantes que el alumnado de niveles educativos inferiores que mostraban un mayor nivel de empatía y disponibilidad social; y con los obtenidos por Caballero, Contini de González, Lacunza, Mejail y Coronel (2017) cuyos resultados señalaban que el alumnado de menor edad contaba con mayor habilidad de autocontrol y liderazgo que el de edad más avanzada, atribuyendo esto a la posibilidad de que el alumnado se rebela contra estas pautas sociales a modo de autoafirmación.

Esta evolución en la competencia sociolaboral del alumnado puede ser fruto del tratamiento de la misma y una mayor profundización dentro del currículo de cada etapa educativa, por lo que, según se avanza dentro del sistema educativo, más peso tienen este tipo de competencias y más se trabajan y entrenan.

Respecto a la situación laboral del alumnado, el alumnado que trabaja cuenta con mayores niveles de satisfacción con la metodología y con el profesorado. Esto puede deberse a la naturaleza laboral de Ceuta, pues la mayor parte de la oferta laboral procede del ámbito educativo (bien por oposiciones, bolsas o planes de empleo de la misma ciudad), por lo que el alumnado que ha trabajado en este área puede tender a empatizar con el profesorado. Así mismo, el alumnado trabajador cuenta con una mejor habilidad para seguir órdenes, una mayor capacidad de análisis y de síntesis, manifestando mayor liderazgo, iniciativa y autonomía.

Estos resultados contradicen a lo manifestado por Caballero, Contini de González, Lacunza, Mejail y Coronel (2017), que establecen que el alumnado desempleado o procedente de hogares con todos los familiares desempleados, es decir, nivel socioeconómico bajo cuentan con mayores habi- lidades sociales de autocontrol y liderazgo que el alumnado con mayor poder adquisitivo.

Por otro lado, estos resultados avalan lo defendido por los estudios de Gnanadevan, Selvaraj y Sirakumar (2017) y de Hosokawa y Katsura (2017) que exponen que tener un empleo mejora el nivel socioeconómico y que un mayor nivel socioeconómico se relaciona con un aumento de habilidad social, mientras que un bajo nivel socioeconómico provoca problemas de conducta en el alumnado, causado por el estrés económico que sufren en sus unidades familiares.

\section{Conclusiones}

Este estudio ha permitido, por un lado, establecer qué alumnado cuenta con mayor nivel de competencia sociolaboral, demostrando que el alumnado con titulaciones superiores (con titulaciones universitarias o ciclos formativos de grado superior) tiene un mayor nivel competencial sobre la disponibilidad social y la tolerancia que el de titulaciones de inferiores rangos.

De igual manera se concluye que el alumnado que cuenta con experiencia laboral o está trabajando manifiesta una mayor satisfacción con la formación y mayores habilidades para seguir órdenes, para el razonamiento numérico, de liderazgo, iniciativa y autonomía, así como una mayor capacidad de análisis y de síntesis.

Como principal limitación, hay que destacar el tamaño tan reducido de la población estudiada, lo que abre nuevas líneas de investigación futuras, aumentando la muestra, relacionando estas habilidades con el nivel socioeconómico y formativo familiar.

\section{Referencias}

1. Caballero, S., Contini de Gonzalez, N., Lacunza, A., Mejail, S. y Coronel, P. (2018). Habilidades sociales, comportamiento agresivo y contexto socioeconómico. Un estudio comparativo con adolescentes de Tucumán (Argentina). Cuadernos de la Facultad de Humanidades y Ciencias Sociales. Universidad Nacional de Jujuy, O(53), 183-203.

2. Gnanadevan, J., Selvaraj, A. y Sivakumar, G. (2015). Influence of Socio Economic Status on Social, Emotional and Behaviour Problem of Students. International Journal of Applied Research, 1(12), 468-471.

3. Hosokawa, R. y Katsura, T. (2017). A longitudinal study of socioeconomic status, family processes, and child adjustment from preschool until early elementary school: the role of social competence. Child and Adolescent Psychiatry and Mental Health, 11(1), 1-28. https://doi. org/10.1186/s13034-017-0206-z

4. Martínez-Rodríguez, F. y Carmona, G. (2010). Test de factores sociopersonales para la inserción laboral de los jóvenes: un instrumento para la evaluación y la formación. Estudios sobre educación, 18 (s-n), 115-138.
5. Navarro-Patón, R., Lago-Ballesteros, J. y Basanta-Camiño, S. (2019). Conductas prosociales de escolares de educación primaria: influencia de los juegos cooperativos. SPORT TK-Revista EuroAmericana De Ciencias Del Deporte, 8(2), 33-37. https://doi.org/10.6018/sportk.401081

6. Reyna, C. y Brussino, S. (2015). Diferencias de edad y género en comportamiento social, temperamento y regulación emocional en niños argentinos. Acta Colombiana de Psicología, 18(2), 51-64. https://doi. org/10.14718/ACP.2015.18.2.5

7. Sánchez-Bolívar, L.; Martínez-Martínez, A. y Parra-González, M. E. (2019). Análisis de las habilidades sociales del alumnado de formación profesional para la entrevista de trabajo. Journal of Sport and Health Research. 11(Supl 1), 127-142.

8. Serrano-Mora, S. M. y Marín-Rodas, C. M. (2015). Competencia social en niños y adolescentes de la ciudad de Bucaramanga (Colombia) según escala Messy. Ciencia y Poder Aéreo, 10(1), 217-228. https://doi. org/10.18667/cienciaypoderaereo.425 